restricted to nurses below 30 years of age, odds ratio (OR) 3.4 (95\% confidence interval [CI] 1.0-12.4). A similar increase in risk for experiencing spontaneous abortions throughout life was found among permanent night-shift workers, OR 4.4 (95\% CI $1.2-16.3$ ), also in this case for nurses below 30 years of age. No increased risk of spontaneous abortions was found among nurses above 30 years of age.

Conclusions The findings suggest that night work may cause spontaneous abortion by disrupting the circadian rhythms, but other unknown mechanisms may also play a role. More studies of night-shift workers considering different age groups are needed to supplement the findings.

\section{PSYCHOSOCIAL JOB STRAIN AND RISK CONGENITAL MALFORMATIONS IN OFFSPRING}

${ }^{1}$ A D L Larsen, 'Hougaard, 'Hannerz, ${ }^{2}$ Thulstrup, ${ }^{3}$ Obel, ${ }^{4}$ Bonde. ${ }^{1}$ National Research Centre for the Working Environment, Copenhagen, Denmark; ${ }^{2}$ Department of Occupational Medicine, Aarhus University Hospital, Aarhus, Denmark; ${ }^{3}$ Department of General Medicine, Institute of Public Health, University of Aarhus, Aarhus, Denmark; ${ }^{4}$ Department of Occupational and Environmental Medicine, Copenhagen University Hos, Copenhagen, Denmark

\subsection{6/oemed-2013-101717.221}

Background Previous studies have shown a relationship between maternal stress during pregnancy and increased risk of malformations in children, eg: cleft lip, palate, neural tube and heart defects. To our knowledge no previous studies has had a jobrelated angle to maternal stress during pregnancy. The present project therefore examines if maternal exposure to psychosocial job strain (high demands and low control) measured by questionnaire early in pregnancy is associated with congenital malformations (all), cardiovascular malformations and malformations in the musculoskeletal system.

Methods We use the Danish National Birth Cohort with more than 100.000 children at baseline. In the present study 60,120 pregnancies are included due to inclusion criteria as: working, pregnant when interviewed, singleton pregnancy and information on exposure, covariates and outcome. Congenital malformations are available from the Danish Medical Birth Register with ICD-10 codes. Analyses are controlled for maternal age, BMI, parity, smoking, alcohol use, type of work, maternal serious disease and gestational age at interview.

Results In total 3,069 cases of malformations were registered in the study population, 582 of these were cases of circulatory malformations and 1,555 of musculoskeletal malformations. Logistic regression analyses showed that high strain was not associated with increased risk of any type of malformations $(\mathrm{OR}=0.99$, CI: $0.85-1.15)$, circulatory $(\mathrm{OR}=1.04$, CI: $(0.75-1.44)$ or musculoskeletal malformations ( $\mathrm{OR}=0.88, \mathrm{CI}: 0.70-1.10)$. The analyses were adjusted for maternal age, parity, smoking, alcohol, maternal BMI, type of work, maternal serious disease, gestational age at interview. Crude analyses did not change the results significantly.

Conclusion The results support the null-hypothesis; that the risk of having a child with congenital malformations is independent of psychosocial job strain in this sample from the Danish National Birth Cohort. As this contradicts previous findings (e.g. bereavement studies, with loss of a child or husband), a discussion regarding exposure and sample size could be beneficial.
Session: Parallel session 2 RICOH: Child behavior and semen quality

\section{MALE REPRODUCTIVE TOXICITY OF PHTHALATES: A CROSS-SECTIONAL STUDY OF TESTOSTERONE AND TOTAL SPERM COUNT IN EUROPEAN AND INUIT POPULATIONS}

${ }^{1}$ I O S Olmer Specht, ${ }^{2}$ Toft, ${ }^{3}$ Jönsson, ${ }^{4}$ Jens Peter. ${ }^{1}$ Copenhagen N, Denmark; ${ }^{2}$ Department of Occupational Medicine, Aarhus, Denmark; ${ }^{3}$ Division of Occupational and Environmental Medicine, Lund, Sweden; ${ }^{4}$ Department of Occupational and Environmental Medicine, Copenhagen N, Denmark

10.1136/oemed-2013-101717.222

Objectives Phthalates are widely used man-made chemicals that in spite of a short half-life in the organism are detectable in urine among more than $95 \%$ of investigated men and women. Phthalates are with varying potency anti-androgens through interaction with several metabolic steps involved in endogenous sex-steroid metabolism. Some cross-sectional studies have shown inverse associations between phthalates and plasma levels of testosterone and some semen characteristics, but the evidence base is limited and results are conflicting. The aim of this study was to examine the hypothesis that phthalates are associated with reduced levels of plasma testosterone and total sperm counts. Methods Spouses of pregnant women from Greenland $(\mathrm{n}=$ 196), Poland $(\mathrm{n}=190)$ and Ukraine $(\mathrm{n}=203)$ were enrolled into the study. We measured six metabolites of di-2-ethylhexyl phthalate (DEHP) and diisononyl phthalate (DINP) in serum and concurrent testosterone, sperm concentration, sperm volume and total sperm count. Analyses were stratified by country as well as analysed across countries.

Results The most abundant metabolite from DEHP namely 5cx-MEPP (mean concentration in serum $2.22 \mathrm{ng} / \mathrm{ml}$ ) was negatively associated with testosterone, sperm volume and total sperm count in the overall analysis after adjustment for country, age, sexual abstinence time and current smoking. Testosterone decreased with $1.08 \% \mathrm{pr} \mathrm{ng} / \mathrm{ml} 5$-cx-MEPP $(\mathrm{p}=0.032)$, volume with $1.59 \%(\mathrm{p}=0.043)$ and total sperm count with $3.47 \%(\mathrm{p}$ $=0.030)$. When analysed by country the association was strongest in Ukraine and Poland, but the inverse relationship between 5 -cx-MEPP and outcomes was observed in all three countries. No significant association between phthalate metabolites and sperm concentration was observed.

Conclusions These results are compatible with a weak antiandrogenic action of the DEHP metabolite 5-cx-MEPP on testosterone and total sperm count. Whether this cross-sectional association reflects causal mechanisms remains to be established.

\section{MOTOR DEVELOPMENT FOLLOWING PRENATAL EXPOSURE TO P,P-DDE AND CB-153: A FOLLOW-UP STUDY OF INUIT AND EUROPEAN CHILDREN AGED 5-9 YEARS}

'B B H Høyer, ${ }^{2}$ Ramlau-Hansen, ${ }^{3}$ Pedersen, ${ }^{4}$ Bonde, ${ }^{1}$ Toft. ${ }^{1}$ Aarhus University Hospital, Aarhus C, Denmark; ${ }^{2}$ Aarhus University, Aarhus C, Denmark; ${ }^{3}$ Centre for Arctic Environmental Medicine, Nuuk, Greenland; ${ }^{4}$ Department of Occupational and Environmental Medicin, Bispebjerg Hospital, Copenhagen NV, Denmark

\subsection{6/oemed-2013-101717.223}

Objectives Both PCB and DDE are lipophilic compounds which bio-accumulate in adipose tissue and cross the placental barrier. Prior studies of the association between prenatal exposure to 
PCB and DDE and child motor development have found contradicting results. The aim of this follow-up study was to examine the association between prenatal exposure to DDE and PCB and motor development and developmental milestones; crawling, standing-up and walking in children in Greenland, Ukraine and Poland.

Methods CB-153 and p,p'-DDE were measured in maternal blood in second or third trimester of pregnancy as a bio-marker of the child's prenatal exposure to the compounds. A total of 1,103 children aged 5 to 9 years were followed up in 20102012. Motor development were measured in terms of the parentally assessed screening tool Developmental Coordination Disorder Questionnaire 2007 (DCDQ'07) and developmental milestones were assessed via parental reports of child age at the first time of crawling, standing up and walking. The association between $\mathrm{PCB} / \mathrm{DDE}$ and motor skills and milestones were analysed by means of linear multiple regression analyses using tertiles of exposure and stratified by country. Both complete case analyses and multiple imputation based analyses were executed. Adjustment were performed for the co-variates; maternal age, maternal smoking during pregnancy, maternal alcohol before pregnancy, maternal education, parity, gestational age at blood sampling, preterm birth, breastfeeding, child sex and child age at interview.

Results We found no associations between prenatal PCB and DDE exposure and developmental milestones or motor skills. Complete case- and multiple imputation based analyses showed adjusted mean differences in motor skills and age at milestones around null, in all three countries.

Conclusions These results on 1.103 mother-children-pairs from the INUENDO cohort in Greenland, Warsaw (Poland) and Kharkiv (Ukraine), indicate no association between in utero PCB/ DDE exposure and developmental milestones and motor skills.

\section{EXPOSURE TO POLYCYCLIC AROMATIC HYDROCARBONS AND SPERM QUALITY OF COKE-OVEN WORKERS}

${ }^{1} \mathrm{~W}$ Y Lin, ${ }^{2}$ Jeng, ${ }^{3} \mathrm{Pan},{ }^{4} \mathrm{M}$ L Yu, ${ }^{4} \mathrm{C}$ Y Dai, ${ }^{1} \mathrm{C} \mathrm{H} \mathrm{Li},{ }^{1} \mathrm{~N}$ C Chang, ${ }^{5}$ Huang, ${ }^{4} \mathrm{M}$ H Hsieh, ${ }^{4} \mathrm{~J} \mathrm{~F}$ Yang, ${ }^{4} \mathrm{C}$ L Wang, ${ }^{6} \mathrm{C}$ F Huang, ${ }^{7}$ Lin, ${ }^{4} \mathrm{H}$ Y Chuang, ${ }^{1} \mathrm{M}$ T Wu, ${ }^{8} \mathrm{C}$ K Ho. ${ }^{1}$ Kaohsiung Municipal Hsiaokang Hospital, Kaohsiung, Taiwan; ${ }^{2}$ School of Community and Environmental Health, College of Health Sciences, Old Do, Norfolk, United States of America; ${ }^{3}$ nstitute of Occupational Safety and Health, Council of Labor Affairs, Taipei, Taiwan; ${ }^{4}$ Kaohsiung Medical University Hospital, Kaohsiung, Taiwan; ${ }^{5}$ Department of Occupational Medicine, Kaohsiung Municipal Hsiaokang Hospital, KMU, Kaohsiung, Taiwan; ${ }^{6}$ Department of Occupational Medicine, Kaohsiung Municipal Ta-Tung Hospital, Kaohsiung, Taiwan; ${ }^{7}$ China Steel Corporation, Kaohsiung, Taiwan; ${ }^{8}$ Department of Health, Kaohsiung City Government, Kaohsiung, Taiwan

\subsection{6/oemed-2013-101717.224}

Objectives The study aimed to assess whether exposure to polycyclic aromatic hydrocarbons (PAHs) alter sperm quality of coke-oven workers.

Methods Personal breathing zone, urine, and semen samples were collected from nonsmoking workers at top-side ovens (high exposure, $\mathrm{N}=16$ ) and side-ovens (low exposure, $\mathrm{N}=20$ ), and administrative staff members serving as the control group $(\mathrm{N}=$ 15). PAH concentrations were analysed by a gas chromatography quadruple spectrometer. Routine semen was analysed by procedures in accordance with the World Health Organization guidelines. Urinary 1-hydroxypyrene (1-OHP) was analysed by HPLC with a fluorescence detector.

Results ANOVA analysis showed a significant difference in urinary 1 -OHP levels $(14.7 \pm 12.9,4 \pm 4.3,0.3 \pm 0.2 \mathrm{~g} / \mathrm{g}$ creatinine respectively, $\mathrm{p}=0.02$ ) between the exposed groups and the control. Mean concentrations of 16 species of PAHs significantly differed among the three groups (Total PAHs $=41620.3 \pm$ $17697.6 \mathrm{ng} / \mathrm{m}^{3}, 19887.6 \pm 1378.1 \mathrm{ng} / \mathrm{m}^{3}, 57.0 \pm 18.1 \mathrm{ng} / \mathrm{m}^{3}$, $\mathrm{p}<0.0001$; Benzo (g,h,i)perylene $=3553.9 \pm 1250.7 \mathrm{ng} / \mathrm{m}^{3}$, $3001.9 \pm 1367.7 \mathrm{ng} / \mathrm{m}^{3}, 2.0 \pm 1.5 \mathrm{ng} / \mathrm{m}^{3}, \mathrm{p}<0.0001, \mathrm{p}=$ 0.067). Asthenospermia was found more frequently in the high exposure group as compared to the control $(37.5 \%$ and $13.3 \%$, respectively, $\mathrm{p}<0.01$ ). The exposed groups had lower percentages of normal morphology as compared with the control group $(14.5 \pm 3.4 \%, 15.0 \pm 3.1 \%$, $34.5 \pm 2.6 \%$ $\mathrm{p}<0.01)$. Multiple regression analysis showed that PAH species positively correlated with abnormality of morphology and motility of sperm.

Conclusion Abnormal morphology of sperm was associated with $\mathrm{PAH}$ concentrations, especially those with heavy molecular weights, e.g. benzo (g,h,i)perylene and benzo (k)fluoranthene.

\section{EXPOSURE TO POLYBROMINATED DIPHENYL ETHERS AND MALE REPRODUCTIVE FUNCTION IN ARCTIC AND EUROPEAN POPULATIONS}

${ }^{1} \mathrm{G}$ T Toft, ${ }^{2}$ Lenters, ${ }^{2}$ Vermeulen, ${ }^{2}$ Heederik, ${ }^{3}$ Thomsen, ${ }^{3}$ Becher, ${ }^{4}$ Giwercman, ${ }^{5}$ Bizzaro, ${ }^{6}$ Manicardi, ${ }^{7}$ Spanó, ${ }^{8}$ Rylander, ${ }^{9}$ Pedersen, ${ }^{10}$ Strucinski, ${ }^{11}$ Zviezdai, ${ }^{12}$ Bonde. ${ }^{1}$ Aarhus University Hospital, Aarhus, Denmark; ${ }^{2}$ Institute for Risk Assessment Sciences, Utrecht University, Utrecht, Nederland; ${ }^{3}$ Department of Chemistry, University of Oslo, Oslo, Norway; ${ }^{4}$ Reproductive Medicine Centre, Skåne University Hospital, Malmö, Sweden; ${ }^{5}$ Department of Life and Environmental Sciences, Polytechnic University of Marche, Ancona, Italy; ${ }^{6}$ Department of Life Science, Università di Modena e Reggio Emilia, Reggio Emilia, Italy; 'Unit of Radiation Biology and Human Health, ENEA Casacia, Rome, Italy: ${ }^{8}$ Division of Occupational and Environmental Medicine, Lund University, Lund, Sweden; ${ }^{9}$ Centre for Arctic Environmental Medicine, Nuuk, Greenland; ${ }^{10}$ Department of Toxicology and Risk Assessment, Warsaw, Poland; ${ }^{11}$ Kharkiv National Medical University, Kharkiv, Ukraine; ${ }^{12}$ Bispebjerg University Hospital, Copenhagen, Denmark

\subsection{6/oemed-2013-101717.225}

Background Animal and a few human studies suggest that polybrominated diphenyl ethers (PBDEs) may affect male reproductive function. The aim of the present study was to evaluate if male reproductive function was associated with serum levels of PBDEs.

Methods We evaluated the effects of environmental exposure to BDE-28, BDE-47 and BDE-153 on reproductive hormones and semen quality, including markers of DNA damage and apoptosis, in 299 men from Greenland, Poland and Ukraine.

Results Adjusted linear regression models indicated that sperm DNA damage measured by the TUNEL assay increased by $0.22 \%$, confidence interval (CI) $0.03 \%$ to $0.42 \%$ for each percentage increase in lipid adjusted BDE-47 concentration, and semen volume decreased by $0.11 \%(0.01 \%$ to $0.19 \%)$ for each percentage increase in BDE-28 exposure.

Conclusions Adverse effects of PBDE exposure on semen volume and sperm DNA damage were observed but other conventional semen parameters and reproductive hormones were not affected. Harmful effects of PBDE exposure on sperm DNA damage is supported by experimental evidence based on other cell types.

\section{PRENATAL BLOOD LEAD LEVEL AND CHILDHOOD NEUROBEHAVIORAL DEFICIT}

${ }^{1} \mathrm{M}$ V Vigeh, ${ }^{2}$ Yokoyama, ${ }^{2}$ Matsukawa, ${ }^{3}$ Shinohara, ${ }^{4}$ Shahbazi, ${ }^{1}$ Ohtani. ${ }^{1}$ National Institute of Occupational Safety and Health, Kawasaki, Japan; ${ }^{2} J$ untendo University Faculty of Medicine, Tokyo, Japan; ${ }^{3}$ Seisen University, Tokyo, Japan; ${ }^{4}$ Tehran University of Medical Sciences, Tehran, Iran

10.1136/oemed-2013-101717.226 